\title{
COMPREHENSIVE SPECTRAL SIGNAL INVESTIGATION OF A LARCH FOREST COMBINING GROUND- AND SATELLITE-BASED MEASUREMENTS
}

\author{
J. M. Landmann ${ }^{\mathrm{a}, *}$, M. Rutzinger ${ }^{\mathrm{a}, \mathrm{b}}$, M. Bremer ${ }^{\mathrm{a}}$, K. Schmidtner ${ }^{\mathrm{b}}$ \\ ${ }^{a}$ Dept. of Geography, University of Innsbruck, 6020 Innsbruck, Austria - \\ (johannes.landmann@student.uibk.ac.at, (martin.rutzinger, magnus.bremer, korbinian.schmidtner)@uibk.ac.at) \\ ${ }^{\mathrm{b}}$ Institute of Interdisciplinary Mountain Research (Austrian Academy of Science), 6020 Innsbruck, Austria - \\ (martin.rutzinger@oeaw.ac.at)
}

Commission VII, WG VII/6

KEY WORDS: Data Fusion, Spectral Signal, Field Spectrometry, Landsat 8, OLI, Classification, Larix decidua

\begin{abstract}
:
Collecting comprehensive knowledge about spectral signals in areas composed by complex structured objects is a challenging task in remote sensing. In the case of vegetation, shadow effects on reflectance are especially difficult to determine. This work analyzes a larch forest stand (Larix decidua MILl.) in Pinnis Valley (Tyrol, Austria). The main goal is extracting the larch spectral signal on Landsat 8 (LS8) Operational Land Imager (OLI) images using ground measurements with the Cropscan Multispectral Radiometer with five bands (MSR5) simultaneously to satellite overpasses in summer 2015. First, the relationship between field spectrometer and OLI data on a cultivated grassland area next to the forest stand is investigated. Median ground measurements for each of the grassland parcels serve for calculation of the mean difference between the two sensors. Differences are used as "bias correction" for field spectrometer values. In the main step, spectral unmixing of the OLI images is applied to the larch forest, specifying the larch tree spectral signal based on corrected field spectrometer measurements of the larch understory. In order to determine larch tree and shadow fractions on OLI pixels, a representative 3D tree shape is used to construct a digital forest. Benefits of this approach are the computational savings compared to a radiative transfer modeling. Remaining shortcomings are the limited capability to consider exact tree shapes and nonlinear processes. Different methods to implement shadows are tested and spectral vegetation indices like the Normalized Difference Vegetation Index (NDVI) and Greenness Index (GI) can be computed even without considering shadows.
\end{abstract}

\section{INTRODUCTION}

Spatial heterogeneity and the combination of geometrical views and shadowing are some of the main obstacles when trying to capture tree spectral signals. From a modeling perspective, $\mathrm{Li}$ and Strahler (1985) found in an illumination analysis that the representation of conifers as cones casting shadow on a contrasting background can be a good approximation to real conditions, concluding that shape, form, and shadowing of objects are important for determining the signal on satellite images. Despite that, Colwell (1974) reports that besides viewing geometry, a much more detailed scale is necessary to predict vegetation canopy reflectance and therefore investigates leaf hemispherical transmittance, leaf area and orientation and vegetation stalk, trunk and limb properties. Practical approaches are often based on field spectrometers positioned above the trees, for example on a $30 \mathrm{~m}$ high mast (Sukuvaara et al., 2007) or the bucket of a lift vehicle (Koch et al., 1990). The problem about these approaches is that they are cost-intensive and it is difficult to tell if the whole tree, more than that or just parts are captured. In this study, we aim at overcoming this issue by a simple geometrical modeling of the larch forest and a linear spectral unmixing of Landsat 8 (LS8) Operational Land Imager (OLI) surface reflectance values, similar to the method as described in Roberts et al. (1998). One endmember component in the spectral unmixing procedure is the understory, which is captured with the Cropscan MSR5, a sensor with OLI-like bands. The other endmember component is the larch trees, the signal we are looking for. Different methods to implement shadows are tested: first, no shadows are considered. In literature, it is reported that the Normalized Difference

${ }^{*}$ Corresponding author
Vegetation Index (NDVI) (Rouse et al., 1974) and the Greenness Index (GI) (Zarco-Tejada et al., 2005) can be calculated without making majors errors (Zhang et al., 2015). The second method includes "binary shadows", which means only a differentiation of sunlit and tree shadow areas. Tree shadow areas are derived from an insolation analysis of a digital forest, consisting of 649 point cloud model trees in $2 \mathrm{~cm}$ resolution. The third method proposed includes the binary shadows, but also a parametrization of terrain shadowing by an inversion of the solar irradiation amount during the satellite overpass. The shadow reflectance in each of the second two approaches can only be estimated and is mainly based on the findings of Zhang et al. (2015) and Leblon et al. (1996). In default of the "true" and pure larch signal, results are compared to two other studies that also deal with larch reflectance. Strengths and shortcomings of all methods are discussed and a short conclusion with respect to future developments is given.

\section{DATA SETS}

Figure 1 presents the test site in Pinnis Valley (Tyrol, Austria). The considered area includes about 12.9 hectares of an east-facing slope at altitudes from roughly $1230 \mathrm{~m}$ to $1510 \mathrm{~m}$ above mean sea level. The lower part is a cultivated grassland with many small parcels used for haymaking and where cattle is grazing periodically. The upper part is a larch forest for which spectral unmixing is performed. Slope steepness is reduced for some parts of the grassland, while most parts exhibit a gradient of about $30^{\circ}$.

On four clear-sky days in summer 2015 (July $15^{\text {th }}$, August $3^{\text {rd }}$, September $1^{\text {st }}$ and October $\left.12^{t h}\right)$, field spectrometer measurements have been taken $\pm 3 \mathrm{~h}$ around LS8 overpasses for each 


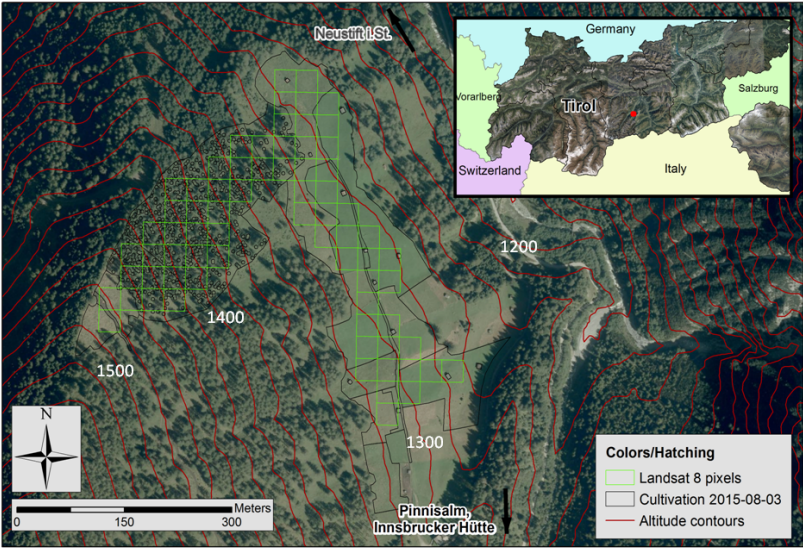

Figure 1: The Pinnis Valley test site (black polygon outlines).

of the unique cultivated grassland areas. On the last three field work days, measurements from the larch understory have been taken, too. The LS8 data contain therefore four scenes, which come from different acquisition paths and rows. The first three images are recorded from Worldwide Reference System (WRS)2 path/row 193/27 with a viewing angle of $2.9^{\circ}$ on the test site and the last one on October $12^{\text {th }}$ is from WRS-2 $192 / 27$ with a viewing angle of $-6.4^{\circ}$. Blue, green, red and Near-Infrared (NIR) bands were downloaded from the United States Geological Survey (USGS) Earth Resources Observation and Science Center (EROS) Science Processing Architecture (ESPA) (United States Geological Survey, 2016) after processing to surface reflectance by using the LS8SR algorithm (United States Geological Survey, 2015b). Together with the surface reflectance bands, the NDVI was downloaded and the surface reflectance GI was calculated employing the ratio of surface reflectance green and red bands.

The collection of ground reference data involves the Cropscan MSR5, a field spectrometer mounted to a $3.5 \mathrm{~m}$ long pole with a footprint opening angle of $28^{\circ}$. The field spectrometer band widths are actually designed to match the Landsat 4/5 Thematic Mapper (TM) instrument (Cropscan Inc., 2015). However, in the meantime band widths have not changed by more than $0.01 \mu \mathrm{m}$, except for a massive band narrowing in the NIR channel of OLI. Table 1 compares the band widths for LS8 and field spectrometer.

\begin{tabular}{|l|c|c|c|}
\hline Band & Field spectrometer $(\mu \mathrm{m})$ & OLI $(\mu \mathrm{m})$ & OLI No. \\
\hline Blue & $0.45-0.52$ & $0.45-0.51$ & 2 \\
Green & $0.52-0.60$ & $0.53-0.59$ & 3 \\
Red & $0.63-0.69$ & $0.64-0.67$ & 4 \\
NIR & $0.76-0.90$ & $0.85-0.88$ & 5 \\
\hline
\end{tabular}

Table 1: Comparison of field spectrometer and Operational Land Imager (OLI) bands.

The band narrowing in the LS8 NIR channel is due to the exclusion of an atmospheric water vapor extinction feature around $0.825 \mu \mathrm{m}$. The comparison of surface reflectance data between Landsat 7 (LS7) and LS8 exhibits that the larger difference between the NIR bands is "not evident" in surface reflectance data and the sensor-to-sensor difference is $1 \%$ to $3 \%$ on average (Flood, 2014). On all four days, 1399 measurements have been taken on the area, about $15 \%$ thereof come from the larch understory. Grassland areas have been sampled spatially regularly with the claim to obtain at least 30 measurements per area. Larch understory has been captured wherever possible as the field spectrometer can - for technical reasons - take measurements only where irradiation is higher than $300 \frac{\mathrm{W}}{\mathrm{m}^{2}}$ (Cropscan Inc., 2001). This is achieved in sunlit patches in the forest.

\section{METHODS}

\subsection{Sensor-to-Sensor Relationship}

At first, an overlay of the satellite raster with the digitized cultivation areas is performed. This results in 34 pixels on the larch forest area and 39 pixels on the cultivated grassland. The latter number is slightly varying with time due to slightly changing cultivation polygon outlines. On the grassland, synthetic satellite pixels are generated from a linear weighted combination of the median values from all cultivation classes contributing to the area of the respective overlaying LS8 pixel. Each of the synthesized pixel values in each band is subtracted from the measured satellite value in the regarding pixel and the channel-wise mean is calculated over all differences. The procedure is repeated for each of the field work days and the mean difference is called "bias" from now on.

\subsection{Digital Forest}

In order to reconstruct the larch forest with its geometrical properties, a representative three-dimensional larch tree shapefile modeled in the way Bremer et al. (2013) describe is used. This shapefile is derived from a Light Detection and Ranging (LiDAR) scan of the forest acquired from the opposite slope in the year 2014. The shape was cut off a subset of about 35 tree shapes at the upper edge of the slope and transformed into a point cloud by sampling the three-dimensional shape with points in $2 \mathrm{~cm}$ distance. The reason for this is that point clouds are computationally much easier to handle than shapefiles. Positions where to plant the virtual trees are determined using an inverse watershed algorithm tree delineation on a $1 \mathrm{~m}$ resolved normalized Digital Surface Model (nDSM) of the area. The procedure includes the application of a Gaussian Filter with search radius $2 \mathrm{~m}$ in order to smooth the $\mathrm{nDSM}$ and a reclassification to exclude vegetation lower than $5 \mathrm{~m}$ (similar approaches can be found in Eysn et al. (2015)). In this manner, 649 trees are identified on the slope and the chosen model tree is planted on each position using a $10 \mathrm{~cm}$ resolved Digital Terrain Model (DTM) as basis for planting. The tree point cloud is turned each time by some degrees such that the forest is not influenced by directional effects. Tree shadows in the forest are calculated using the Laserdata LIS (LIS) SADO (System for the Analysis of Discrete Surfaces; Böhner et al. (1997); Rieg et al. (2014)) insolation multiscale module. For each of the LS8 overpass minutes, the direct mean insolation on a $1 \mathrm{~cm}$ resampled DTM is calculated and hard shadow areas are assigned where the result of this calculation is zero. The larch area fraction per LS8 pixel is determined by an overlay of the vertically projected digital larch trees with the pixel borders. However, as the single trees have lots of holes and single features and the procedure has to be performed with vector data, the method becomes computationally very intensive. In order to keep it as easy as possible, larch trees are for this analysis represented by circles each of which has exactly the same area as a vertically projected tree.

\subsection{Larch Signal Extraction}

The main method applied in this work is a linear spectral unmixing based on an altered, extended and rearranged version of the unmixing equation presented in Roberts et al. (1998) (equation 1).

$$
\rho_{\text {total }, \lambda, i}=\left(A_{L, i} \cdot \rho_{L, i, \lambda}\right)+\left(A_{U, i} \cdot\left(\rho_{U, \lambda *}^{f}-b\right)\right)+\epsilon
$$

where $\quad \rho_{t o t a l, \lambda, i}=$ surface reflectance of satellite pixel $\mathrm{i}$ 


\author{
at band $\lambda$ (unitless) \\ $A_{L, i}=$ larch area fraction of pixel i (unitless) \\ $\rho_{L, i, \lambda}=$ larch reflectance for pixel i (unitless) \\ $A_{U, i}=$ understory area fraction for pixel i (unitless) \\ $\rho_{U, \lambda^{*}}^{f}=$ understory reflectance measured with field \\ spectrometer at band $\lambda^{*}$ (unitless) \\ $b=$ bias: field spectrometer minus satellite (unitless) \\ $\epsilon=$ error term
}

The letter $\rho$ is used in general for reflectance values, but also counts for the vegetation indices NDVI and GI in this study, as they are also unmixed directly. The two biggest assumptions with this approach are the exclusion of non-linear processes, such as multiple scattering in the forest, and that the field spectrometer measurements mirror the natural variability of the class. $\epsilon$ is generally set to zero, consequently.

Equation 1 does not contain any shadow term and is therefore used to determine the larch reflectance with the "no shadows" method. For the shadow implementation, it has to be determined by which proportion the sunlit reflectance is reduced when shaded. The following values are introduced as Band Shadow Reduction Factors (BSRFs) and are estimated based on the work of Zhang et al. (2015) and Leblon et al. (1996).

- Blue: 0.1

- Green: 0.05

- Red: 0.1

- NIR: 0.2

They are applied to equation 2 in order to calculate the larch signal when binary shadows are considered.

$$
\begin{array}{r}
\rho_{L, i, \lambda}=\frac{\rho_{\text {total }, \lambda, i}-\left(A_{s, i} \cdot B S R F_{\lambda} \cdot\left(\rho_{U, \lambda *}^{f}-b\right)\right)}{A_{L, i}} \\
-\frac{\left(1-A_{L, i}-A_{s, i}\right) \cdot\left(\rho_{U, \lambda *}^{f}-b\right)}{A_{L, i}}
\end{array}
$$

where $\quad A_{s, i}=$ shadow fraction per pixel (unitless)

$B S R F_{\lambda}=$ BSRF for the respective band (unitless)

The terrain shadowing method includes both the hard larch tree shadows and an attempt to parametrize the shadows caused by the terrain, assuming that there is not a distinction between hard and no shadow only, but that there are continuous levels of shadow (equation 3).

$$
\tilde{A}_{s, i}=\frac{1}{n} \sum_{k, j} 1-\frac{I_{k, j}-I_{\min }}{I_{\max }-I_{\min }}
$$

where $\quad \tilde{A}_{s, i}=$ shadow fraction for satellite pixel i (unitless)

$n=$ number of DTM cells within a satellite pixel

$k, j=$ line, column coordinates of the shadow raster

$I_{k, j}=$ mean solar irradiance during one second at the satellite overpass $\left(\frac{W}{m^{2}}\right)$

$I_{\max }, I_{\min }=$ maximum and minimum of $I$ on the DTM area $\left(\frac{W}{m^{2}}\right)$
The overall shadow fraction on a satellite pixel is calculated by an averaging of all hard shadows and all parametrized shadow values. The same equation 2 is used, but $\tilde{A}_{s, i}$ is inserted instead of $A_{s, i}$.

\section{RESULTS}

Table 2 informs about the mean differences between field spectrometer synthesized pixels and original OLI reflectance values. All differences are below $0.6 \%$, with only some exceptions. The visible channels on July $15^{\text {th }}$ exhibit differences up to $1.7 \%$ and the NIR channel on July $15^{\text {th }}$ and October $12^{\text {th }}$ differs by $3.3 \%$ and $5.6 \%$ on average. Applying these results to the spectral unmixing, the corrected OLI larch signal can be derived. Figure 2 shows the results for August $3^{\text {rd }}$, comparing the three shadow implementation methods. Boxes show the median values (red line), first and third quartile (boxes) and 1.5 times the interquartile range (whiskers). Every value beyond the latter range is marked as a cross and printed numbers are the median values.

For the "no shadows" method, all extracted larch surface reflectance median values are below zero. A large variability - as determined by the range covered by the whiskers - can be observed for the NIR band, even though in the negative range. Vegetation indices, however, are positive with a median value of 0.84 for the NDVI and 1.23 for the GI. Major changes arise when the binary shadow method is employed. Surface reflectance becomes positive without exception and exhibits a $2 \%$ reflectance in each visible band and $17 \%$ in the NIR band. The variability in the NIR and green channel as well as in the NDVI is reduced to roughly the half of what is found when shadows are not considered. In contrast to that, the NDVI and GI median values show only relatively small changes. The NDVI is reduced to 0.75 and the GI increases to 1.99. Applying the terrain shadow method, changes observed are much smaller as compared to switching from no shadows to the binary shadow method. Variabilities are again reduced, especially in the green and NIR band.

Figure 3 depicts the temporal evolution of the extracted median larch reflectances when derived with the three shadow methods presented. It can be clearly seen that the method without considering shadows is not able to reproduce physically reasonable results as all found reflectances are negative. This is true for all dates. What cannot be revealed by the comparison of the boxplots in Figure 2 is that also the signal from the binary shadows method decreases with time (dashed lines). Especially October $12^{\text {th }}$ is a problematic case, where in fact the blue and green channels are still positive, while reflectance in the red range is equal to zero. The terrain shadow parametrization method, however, does not show this behavior. Values for the RGB range of the spectrum remain constant or even show a slight increase over time, but do not become zero or negative. The NIR reflectance does not reduce as much as in the binary shadow case on October $12^{t h}$, while the red reflectance even increases slightly.

\begin{tabular}{|l|r|r|r|r|}
\hline Date (2015) & SR B & SR G & SR R & SR NIR \\
\hline $07-15$ & 0.0122 & 0.0105 & 0.0165 & -0.0333 \\
$08-03$ & 0.0018 & -0.0037 & -0.0021 & 0.0014 \\
$09-01$ & 0.0052 & 0.0029 & 0.0021 & -0.0022 \\
$10-12$ & -0.0024 & -0.0051 & -0.0008 & -0.0563 \\
\hline
\end{tabular}

Table 2: Mean difference between field spectrometer and satellite values when comparing the spectrally synthesized field spectrometer pixels with the original Operational Land Imager (OLI) values. Satellite reflectances are subtracted from those synthesized. "SR" means "surface reflectance" and "B", "G", "R" and "NIR" mean "blue", "green", "red" and "near-infrared", respectively. 

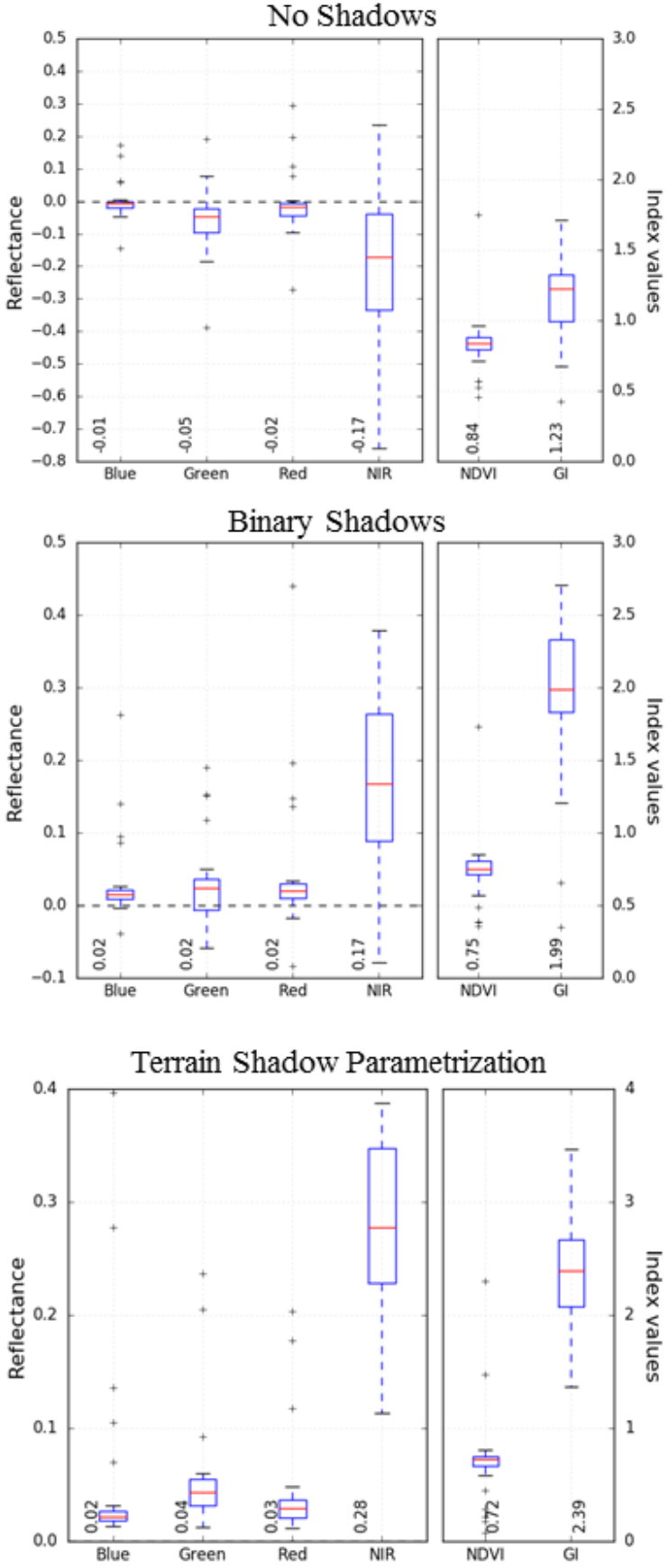

Figure 2: Comparison of the three shadow implementation methods for the corrected larch signal on August $3^{\text {rd }}, 2015$.

As the terrain shadow parametrization method is the only method producing continuously physically reasonable results, Table $3 \mathrm{com}$ pares the found larch reflectance values with this method with values from two other studies.

Kajisa et al. (2007) investigated the spectral trajectory of a larch forest stand (Larix kaempferi, Japanese larch) in the experimental forest of Kyushu University, Hokkaido, Japan, with increasing stand volume for Landsat 5 TM on Day of Year (DOY) 266. For this, they fitted an exponential decrease function to distinct data points which results in an asymptotic reflectance value for an increasing stand volume. The values found in this table are rounded numbers of the correlation coefficients as given in the

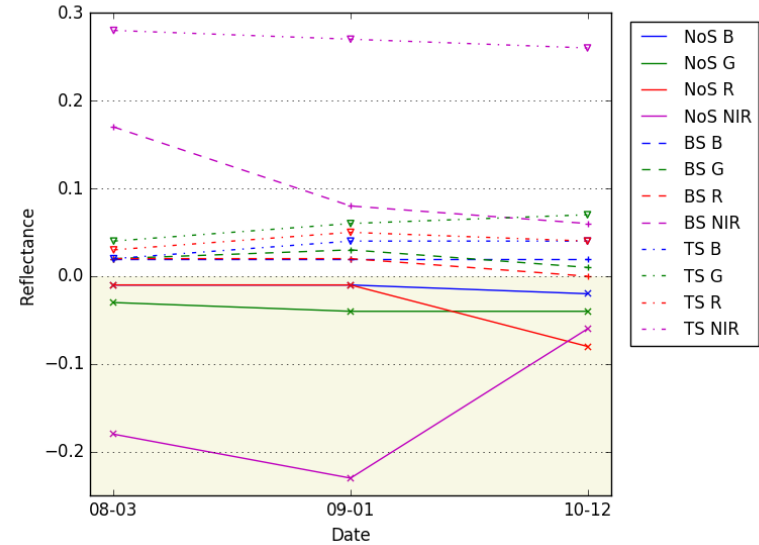

Figure 3: Temporal development of the extracted larch reflectances without shadows considered, with binary shadows considered and the terrain shadow parametrization applied. NoS stands for the no shadows-method, BS means binary shadows and TS is short for terrain shadows. The physically not reasonable range is shaded in yellow.

\begin{tabular}{|l|l|l|l|l|l|}
\hline $\begin{array}{l}\text { Band } / \\
\text { Index }\end{array}$ & $\begin{array}{l}\text { OLI } \\
(08- \\
03)\end{array}$ & $\begin{array}{l}\text { OLI } \\
(09- \\
01)\end{array}$ & $\begin{array}{l}\text { OLI } \\
(10- \\
12)\end{array}$ & $\begin{array}{l}\text { Kobayashi } \\
\text { et al. } \\
(2007) \\
(\text { ETM+) }\end{array}$ & $\begin{array}{l}\text { Kajisa et } \\
\text { al. (2007) } \\
(\mathrm{TM})\end{array}$ \\
\hline B & 0.02 & 0.04 & 0.04 & - & 0.02 \\
G & 0.04 & 0.06 & 0.07 & - & 0.03 \\
R & 0.03 & 0.04 & 0.04 & 0.04 & 0.02 \\
NIR & 0.28 & 0.27 & 0.26 & 0.22 & 0.22 \\
NDVI & 0.72 & 0.59 & 0.57 & $0.69^{*}$ & $0.83^{*}$ \\
GI & 2.39 & 2.33 & 3.10 & - & $1.50^{*}$ \\
\hline
\end{tabular}

Table 3: Comparison of the found values for the terrain shadow parametrization method on August $3^{\text {rd }}$ (DOY 212), September $1^{\text {st }}$ (DOY 244) and October $12^{t h}$ (DOY 285), 2015, with other studies. Compared is the median of the bias-corrected values for Landsat 8 Operational Land Imager (OLI) with the values found by Kobayashi et al. (2007) for Landsat 7 (LS7) Enhanced Thematic Mapper Plus (ETM+) and Larix gmelinii on DOY 170 and the values found by Kajisa et al. (2007) for Landsat 5 Thematic Mapper (TM) and Larix kaempferi on DOY 266. An asterisk means that the value was calculated from the found band spectral values in the study.

publication. Kobayashi et al. (2007) performed a radiative transfer analysis including an overpass of Landsat 7 ETM+ on DOY 170 for an area in Siberia with Larix gmelinii (Siberian larch). As no details are given in the publication, values were read from the diagrams, here. The overall agreement between the findings is strongly dependent on the DOY considered. If the Root Mean Square Error (RMSE) is taken into account as a measure of agreement between the reflectances found, August $3^{\text {rd }}$ fits best with the measurements found by the two other studies, even though for example both September $1^{\text {st }}$ and October $12^{\text {th }}$ are closer to DOY 266, where the data from Kajisa et al. (2007) are measured. The smallest difference with a deviation of only $4 \%$ is found for the NDVI on August $3^{\text {rd }}$ compared to the NDVI found by Kobayashi et al. (2007) for ETM+. 


\section{DISCUSSION}

\subsection{Field Spectrometer Measurements}

The field spectrometer values undergo a correction in two steps: first, radiation at the top of the sensor passes an ideal diffusely transmitting opal glass and is corrected for the sun zenith angle. The second correction includes the application of internal correction factors for imperfect irradiance diffusion of the opal glass and small reflectance properties inside the module tube. These latter correction values come from a pre-delivery factory calibration and should not be altered by the user (Cropscan Inc., 2001). In the former case, however, small inaccuracies in horizontal adjustment of the radiometer can cause errors in reflectance measurement. As errors can reach high values, it is suggested not to use the field spectrometer when the sun zenith angle is bigger than $60^{\circ}$ (Cropscan Inc., 2001). This requirement has been exactly fulfilled as the maximum sun zenith angle for which measurements are taken is $59.2^{\circ}$ on October $12^{\text {th }}$. The lowest sun zenith angle is $25.8^{\circ}$ on July $15^{\text {th }}$. Figure 4 shows how the measured reflectance varies with different inaccuracies in the spectrometer leveling at different sun zenith angles. Errors can reach

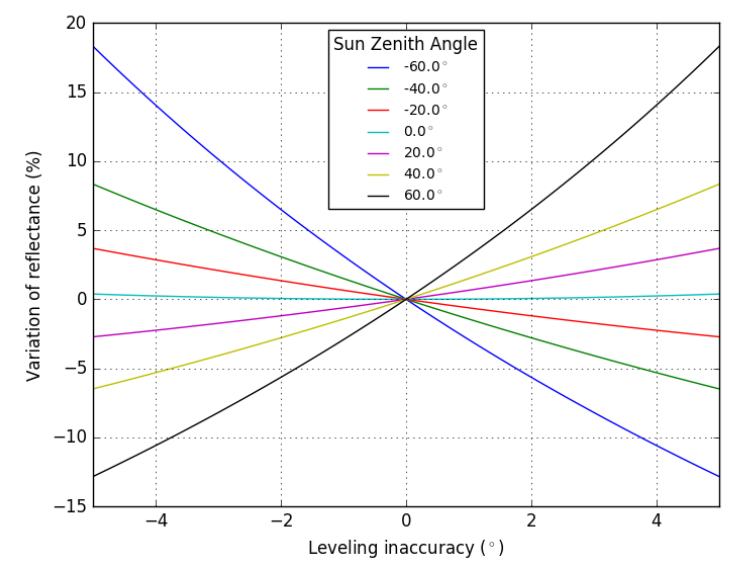

Figure 4: Sensitivity of the found reflectance to inaccuracies in leveling of the field spectrometer. Positive leveling inaccuracies mean a clockwise rotation of the field spectrometer when its front is positioned towards the sun, negative values indicate a counterclockwise rotation.

up to an $18 \%$ relative change of the reflectance measured for a sun zenith angle of $\mp 60^{\circ}$ and a $\mp 5^{\circ}$ horizontal adjustment error. It can be seen that the errors are only relevant some values in the RGB range as their values mostly do not exceed $5.5 \%$ and the error-relevant digits are therefore not considered in this study. However, as the NIR channel clearly exceeds $10 \%$, the errors become most relevant for this channel and increase over course of time. This could possibly explain the bigger difference between NIR values on October $12^{\text {th }}$, which reaches $5.6 \%$. Considering all median NIR measurements for the respective cultivation areas, the $\pm 5 \%$ error could explain a $6.2 \%$ absolute error for reflectance measurements. It remains unsolved with this explanation why for example the reflectance difference on July $15^{\text {th }}$ also reaches $3.3 \%$. Besides the higher differences in the NIR band, the differences are in the range of or below the difference of $2 \%$ that Flood (2014) found for a LS7 to LS8 surface reflectance sensor cross-calibration.

A means to improve the determination of sensor-to-sensor relationship could be the introduction of Spectral Band Adjustment Factors (SBAFs) as described in Chander et al. (2013). They used the ratio of sensor relative spectral responses in order to calculate SBAFs, which relate the reflectance measurements of one sensor with those of another one on pseudoinvariant test sites. However, the approach cannot be put into practice in this study as the exact relative spectral response is not known for the field spectrometer.

\subsection{Viewing Geometry}

On a more general level, it has to be accounted for the fact that the cultivation area itself belongs to a slope and is not perfectly suited for a sensor-to-sensor calibration. Other studies suggest to use deserts or at least flat and homogeneous areas (confer e.g. Czapla-Myers et al., 2015; Mishra et al., 2014). The slope has been used nevertheless, because it is very close to the larch forest and other sites suited for calibration are not reachable within some kilometers distance. This is a logistic problem as measurements have to be temporally close to satellite overpasses. In this context it has to be addressed, too, that field spectrometer and satellite do not have the same viewing geometry. The LS8 images claim to be taken in "nadir" view (as adopted from the image metadata), the Pinnis test site is $2.4^{\circ}$ for WRS-2 193/27 and $-6.9^{\circ}$ for WRS-2 $192 / 27$ away from the absolute nadir view though. Figure 5 gives an impression of the two satellite views on the test site. Apart from the fact that the viewing direction is

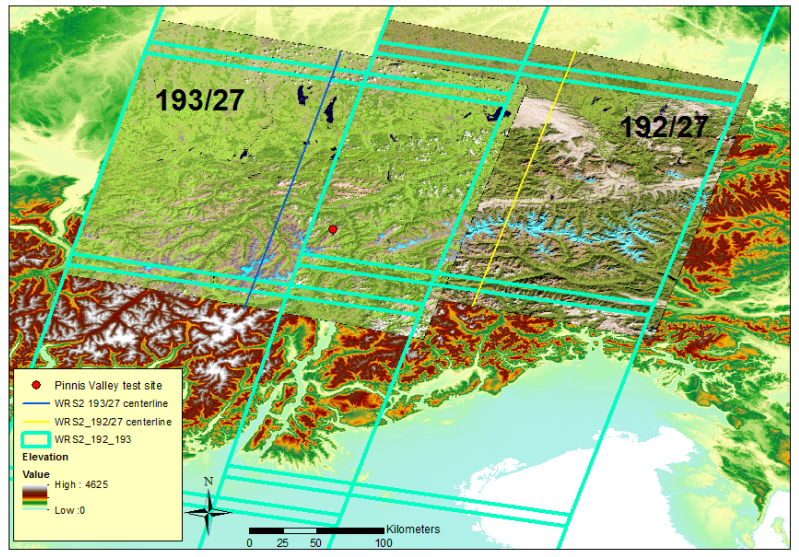

Figure 5: The Pinnis test site is located opportune on the overlapping area of two descending Worldwide Reference System (WRS)-2 paths, shown in blue. As can be identified from the centerlines of the images acquired in row 27 , the center of path 193 is closer to the test site than that of path 192. Also the view direction switches between the two acquisition positions.

turned $180^{\circ}$ on the horizontal plane, the viewing zenith angles are low with $2.4^{\circ}$ and $-6.9^{\circ}$ (confer the distance of the scene centerlines to the test site). Holben and Fraser (1984) support the assumption that the angle difference of $2.4^{\circ}$ and $-6.9^{\circ}$ for one scan does not matter by a modeling of canopy red and NIR responses to change in viewing angle. Suits (1973) states that the cause of the azimuthal variation in reflectance is determined by the observed proportions of reflection and transmission of the specular flux field by the vertical components of the vegetation. As for example the leaf direction distribution of a normal pasture is random, the actual azimuthal dependency of reflectance should depend more on the sun azimuth angle. Gao et al. (2014) investigated angular reflectance for different types of canopies and found that for the small swath width of Landsat (TM in this case, but OLI has the same value) and a grass canopy the difference of reflectance from nadir even to the view zenith angle of $-6.9^{\circ}$ is below one percent. An additional effect on the sloped terrain is, however, that the distribution of grass leaf directions is indeed 
also random, but the terrain is inclined. The effects of this combination should actually be included in the LS8 preprocessing. As topography varies also on much smaller scales than the $30 \mathrm{~m}$ resolution of the correction Digital Elevation Model (DEM) used in the processing (United States Geological Survey, 2015a), the effects of small-scale slope variation are still to be investigated.

\subsection{Larch Signal Extraction}

Comparison of the found larch reflectance with the terrain shadow parametrization method with the two studies Kobayashi et al. (2007) and Nakaji et al. (2008) shows that values are equal in range, even though many deviations exceed $10 \%$ relative change. Differences can be explained by the use of different sensor (ETM+ and TM), different measurement dates, different larch species and different climatic conditions.

Concerning the performance of the applied shadow methods, an assessment which of the three shadow methods is the most accurate one is only possible on the basis of comparison to the two studies mentioned above and the temporal evolution of larch reflectance medians extracted with the three shadow methods. What can be proven is that shadows have to be considered, when the larch tree surface reflectance shall be derived. The reason is that all extracted band reflectances without considering shadows are negative. This is physically not meaningful and, consequently, the method is not applicable for deriving band reflectance. By contrast, the comparison of the binary and the terrain shadow parametrization method demands a closer examination. Only the zero red reflectance on October $12^{\text {th }}$ gives a hint on the reliability of the binary shadow method, because all other values seem to agree well with the findings by Kobayashi et al. (2007) and Nakaji et al. (2008). Assuming that also this method could be marked up as a big step towards the "truth", but not the most accurate one, the terrain shadow method is the most plausible approximation to the true and overall larch signal. Due to the fact, however, that BSRFs have been read from diagram values and depend in reality on the object characteristics considered (grass, soils...), it is obvious that also the terrain shadow parametrization method is a limited approach. Moreover, the terrain shadow is just parametrized and based on a bilinear interpolation of a $1 \mathrm{~m}$ resolved DTM, which is also just a model approach. Generally, the method of spectral unmixing might be easier applicable to spruce trees or other "dense" trees, as they grow in more regular shapes, have a relatively higher Leaf Area Index (LAI) and allow less sunlight to penetrate the crown (Lio, 2014). Nevertheless, it could be shown that even for a complex-structured larch forest the unmixing delivers solid results. The vegetation indices NDVI and GI found to be in the range of values found by Kajisa et al. (2007) even without considering shadows at all. This could be a hint that these vegetation indices are relatively unaffected by shadow, as shown for the NDVI and GI in Zhang et al. (2015).

If not only the median of the found larch reflectance is considered, the inter-pixel variability is still very high, especially in the important NIR band. Still, absolute NIR reflectance variability is generally higher as could be observed for the field spectrometer measurements on the grassland area. This variability for trees might come on the one hand from the natural reflectance variability of the real trees as captured on the LS8 image. On the other hand it can also be a result of different phenological states of the single trees and mutual shadowing. As the overall tree signal as a combination of sunlit and shadowed parts is captured, also mutual shadowing of crowns becomes an important factor (Li and Strahler, 1992). The found results are not suitable for an exact species identification as there are simply too few channels involved. Nevertheless, they can definitely be used for classification purposes. For example, they could serve as class centers and variability estimates for a supervised classification. The classification accuracy, for example compared to a manual training, should be explored in further analyses. However, coming closer to a species-specific spectral signature, the inclusion of more spectral bands and more viewing directions would be necessary.

Concerning the geometrical approach with 3D tree shapes applied here, the main advantages of this method are the simplicity and computational effectiveness. As mentioned in section $1, \mathrm{Li}$ and Strahler (1985) used an even simpler approach with cones representing conifers casting shadow on a contrasting background. The fact that all of the attributes they found important - shape, form and shadowing - are considered also in this study reflects the feasibility of the method. Of course, the larch tree fraction calculation with the help of circles representing the single trees is a heavy assumption. Especially at pixel borders inaccuracies can happen as the circle diameter is not as large as the tree diameter. However, as the effect occurs from both sides, it evens out.

By the example of the NIR channel on August $3^{\text {rd }}$, Figure 6 reveals that there are many outliers found in the larch spectral unmixing procedure. They are illustrated by displaying the modi-

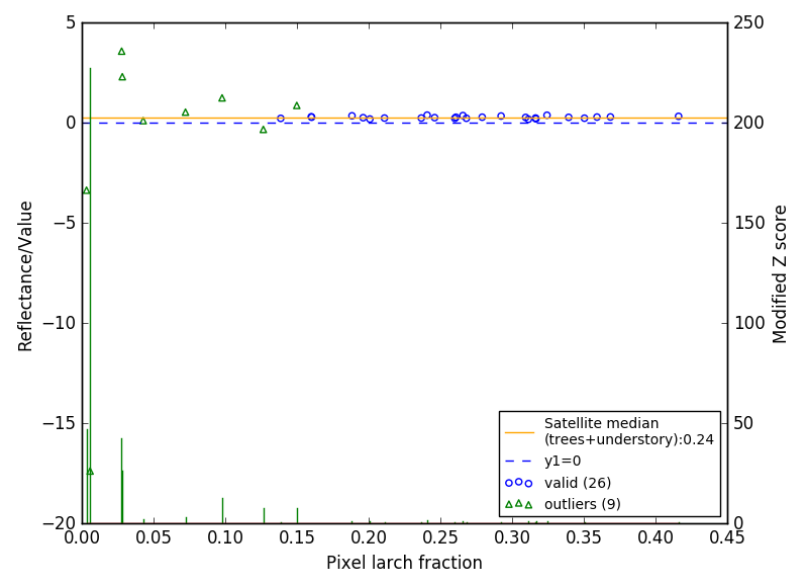

Figure 6: Many larch Near-Infrared (NIR) outliers are found when the pixel larch fraction is smaller than 0.1 .

fied $\mathrm{Z}$ score of the dataset on the right-hand $\mathrm{Y}$ axis, a measure to mathematically determine outliers as presented by Iglewicz and Hoaglin (1993). The suggested threshold of 3.5 is chosen to mark a data point as outlier (green triangles). A common feature for almost all outliers calculated using the modified $\mathrm{Z}$ score is that they appear at larch area fractions smaller than 0.1. In general, after the spectral unmixing the larch reflectance should be constant, regardless of the larch tree area assumed on the pixel. Slight increases when the larch area fraction becomes smaller are possible due to the fact that e.g. mutual shadowing effects become smaller. However, reflectances exceed the value of 1 and fall below zero in this case, which is physically not possible. Leaving out these values shows a better independence of the found larch signal from the assumed larch fraction. Consequently, it can be assumed that there is an issue with most of the pixels with a small larch fraction and that is why the unmixing algorithm fails for them. To improve the unstable behavior of the signal extraction, the quality of the tree delineation algorithm should be investigated in further studies. A more realistic tree shape representation would also be desirable, even though the computational effort is much higher then. 
One general issue in this study is, however, that the results cannot be proven and there is - despite comparisons - no reference and "truth" value given. Chosen BSRFs are just estimates and therefore arbitrary. Even though the found reflectances are roughly coherent with findings of related work, a simulation of different BSRF combinations is desirable. Another approach could be measuring with a field spectrometer which is also able to deal with shadow reflectances.

\section{CONCLUSIONS AND OUTLOOK}

This work investigates the spectral signal of a larch forest stand (Larix decidua Mill.) in Pinnis Valley (Tyrol, Austria). The main goal is to extract the larch tree spectral signal in Landsat 8 (LS8) Operational Land Imager (OLI) visible and Near-Infrared (NIR) surface reflectance channels, using combined ground- and satellite-based measurements. The described procedures for achieving this goal are computationally inexpensive and based on a linear spectral unmixing approach. The ground data include measurements of the larch forest understory with the Cropscan Multispectral Radiometer with five bands (MSR5) with OLI-like channels, simultaneously to satellite overpasses on August $3^{\text {rd }}$, September $1^{s t}$ and October $12^{t h}, 2015$. In order to account for sensor-to-sensor differences, the relationship between ground- and satellite measurements is investigated on these three days, and July $15^{\text {th }}$, additionally: field spectrometer values have been taken for each of the cultivated parcels and based on a spatial overlay, LS8 pixels are generated synthetically from these measurements. The mean difference between field spectrometer and satellite for the respective bands is used to correct the field spectrometer values for the mean ground-to-satellite difference in the unmixing procedure. All found differences in the visible range of the spectrum are small as compared to sensor-to-sensor differences that other studies found (cf. e.g. Flood, 2014), except for the visible channels on July $15^{\text {th }}$ and NIR values on July $15^{\text {th }}$ and October $12^{\text {th }}$. Some of these inaccuracies can be explained by errors in spectrometer horizontal alignment at high sun zenith angles.

For the larch signal extraction procedure a digital forest has been built, consisting of 649 three-dimensional modeled trees extracted from a $1 \mathrm{~m}$ resolved normalized Digital Surface Model (nDSM) of the area, employing an inverse watershed tree delineation algorithm. The tree area per Landsat pixel is derived from the area of perpendicularly projected circular model trees. A comprehensive shadow modeling has been performed by testing three shadow methods. Hard shadows were calculated from tree point clouds in $2 \mathrm{~cm}$ resolution for each of the satellite overpasses and ground shadow rasters were derived from that. The larch signal was derived for three cases: no shadows considered, only hard shadows from the trees (binary shadows) and a combination of hard shadows and a terrain shadowing parametrization. Without considering shadows, the only usable signals have been found the Normalized Difference Vegetation Index (NDVI) and Greenness Index (GI) with values at least in the order of magnitude the found indices by Kajisa et al. (2007) including shadows. This could indicate that, indeed, these indices are quite "shadow-resistant", even though this hypothesis has to undergo further testing. The surface reflectance channels show negative values without exception. For the binary shadow method, a big step forward could be made: all reflectances found with this approach are positive and in a physical meaningful range, except for the red band on October $12^{\text {th }}, 2015$, which is zero. A further improvement is reached with parametrizing the terrain shadowing by an inversion of the radiation amount that is received during the satellite overpasses. The found larch signals with this method are positive without exception and agree well with the findings from other studies (cf.
Kobayashi et al., 2007; Kajisa et al., 2007). Uncertainties of the procedure remain principally in the inexact tree shape representation, missing comprehensive and distributed measurements of the underground and estimated parameters for a reduction from sunlit to shadow reflectance.

In future studies, the workflow presented can be further developed. Even though it will be computationally much more expensive, latest advances in technology use laser scanners mounted on Unmanned Aerial Vehicles (UAVs) (cf. e.g. Wallace et al., 2012). With this method it is possible to scan the whole forest in much higher precision and within shorter time. Laser scanning the forest from above and not from the opposite slope allows further to model tree shapes much more exact without missing backsides. Every tree's own shape is depicted and hence a fully determined geometrical view of the forest with better estimates of larch area per pixel and shadow fractions can be established. This geometrical view can then also include sensor and sun geometry which enables a better quantitative estimation of the influences that for example a change in acquisition path has. Mounting a field spectrometer on a UAV would further allow to capture the forest and underground signal spatially referenced at a higher resolution. Reflectance values could be captured within a much shorter range of time around the satellite overpass, coming closer to the real viewing and sun. In order to compare results and supply real "truth" data, a radiative transfer modeling of the forest could be performed in order to compare the two datasets (costeffective method presented here and intensive radiative transfer modeling).

With respect to the satellite resolution, big steps towards better results can be made with the Sentinel 2A (SEN-2A) satellite. Compared to LS8, its ground sampling distance is $10 \mathrm{~m}$ and therefore three times higher. Furthermore, at the latitude of the Pinnis test site the revisiting time will be reduced to two or three days together with Sentinel-2B whose launch is planned for mid of 2016 (European Space Agency, 2016).

Summing up, this work contributes to the field of ground truth data collection and comparison in remote sensing as it supplies a comparison of computationally inexpensive approaches to extract the larch tree spectral signal. Ground to satellite sensor differences are found small in general, nevertheless field spectrometer values have been adjusted to fit the satellite values. The method that accounts for both the larch hard shadows and a terrain shadow parametrization is found most accurate for the signal extraction. However, future work has to be done in order to investigate error sources quantitatively and set up a database for comparison.

\section{ACKNOWLEDGEMENTS}

We would like to thank all field work volunteers for the great support: Carina Draschl, Robert Niederheiser and Cindy Rabe. Further, we would like to thank Frederic Petrini-Monteferri from Laserdata $\mathrm{GmbH}$, Innsbruck, for supplying the insolation module.

\section{References}

Böhner, J., Köthe, R. and Trachinow, C., 1997. Weiterentwicklung der automatischen Reliefanalyse auf der Basis von digitalen Geländemodellen. Göttinger Geographische Arbeiten 100, pp. 3-21.

Bremer, M., Rutzinger, M. and Wichmann, V., 2013. Derivation of tree skeletons and error assessment using LiDAR point 
cloud data of varying quality. $\{$ ISPRS $\}$ Journal of Photogrammetry and Remote Sensing 80, pp. $39-50$.

Chander, G., Mishra, N., Helder, D., Aaron, D. B., Angal, A., Choi, T., Xiong, X. and Doelling, D. R., 2013. Applications of spectral band adjustment factors (SBAF) for cross-calibration. Geoscience and Remote Sensing, IEEE Transactions on 51(3), pp. 1267-1281.

Colwell, J. E., 1974. Vegetation canopy reflectance. Remote Sensing of Environment 3(3), pp. 175-183.

Cropscan Inc., 2001. Multispectral Radiometer User's Manual. Cropscan Inc. No explicit author given.

Cropscan Inc., 2015. Cropscan Inc. Web Page. http:// cropscan.com/msr.html. Accessed: 2015-10-04.

Czapla-Myers, J., McCorkel, J., Anderson, N., Thome, K., Biggar, S., Helder, D., Aaron, D., Leigh, L. and Mishra, N., 2015. The ground-based absolute radiometric calibration of Landsat 8 OLI. Remote Sensing 7(1), pp. 600-626.

European Space Agency, 2016. Sentinel-2b new launch date announced. http://www.esa.int/Our_Activities/ Observing_the_Earth/Copernicus/Sentinel-2/New_ launch_date_set_for_Sentinel-2A. Accessed: 201604-04.

Eysn, L., Hollaus, M., Lindberg, E., Berger, F., Monnet, J.-M., Dalponte, M., Kobal, M., Pellegrini, M., Lingua, E., Mongus, D. et al., 2015. A benchmark of LiDAR-based single tree detection methods using heterogeneous forest data from the Alpine space. Forests 6(5), pp. 1721-1747.

Flood, N., 2014. Continuity of reflectance data between Landsat7 ETM+ and Landsat-8 OLI, for both Top-of-Atmosphere and surface reflectance: A study in the Australian landscape. Remote Sensing 6(9), pp. 7952-7970.

Gao, F., He, T., Masek, J. G., Shuai, Y., Schaaf, C. B. and Wang, Z., 2014. Angular effects and correction for medium resolution sensors to support crop monitoring. Selected Topics in Applied Earth Observations and Remote Sensing, IEEE Journal of 7(11), pp. 4480-4489.

Holben, B. and Fraser, R. S., 1984. Red and near-infrared sensor response to off-nadir viewing. International Journal of Remote Sensing 5(1), pp. 145-160.

Iglewicz, B. and Hoaglin, D. C., 1993. How to detect and handle outliers. Vol. 16, Asq Press.

Kajisa, T., Murakami, T., Mizoue, N. and Yoshida, S., 2007. Differences in spectral trajectory with stand volume development between Japanese larch and Japanese oak in Hokkaido, Japan. Journal of forest research 12(6), pp. 435-441.

Kobayashi, H., Suzuki, R. and Kobayashi, S., 2007. Reflectance seasonality and its relation to the canopy leaf area index in an eastern Siberian larch forest: Multi-satellite data and radiative transfer analyses. Remote Sensing of Environment 106(2), pp. $238-252$

Koch, B., Ammer, U., Schneider, T. and Wittmeier, H., 1990. Spectroradiometer measurements in the laboratory and in the field to analyse the influence of different damage symptoms on the reflection spectra of forest trees. International Journal of Remote Sensing 11(7), pp. 1145-1163.

Leblon, B., Gallant, L. and Granberg, H., 1996. Effects of shadowing types on ground-measured visible and near-infrared shadow reflectances. Remote Sensing of Environment 58(3), pp. $322-328$.
Li, X. and Strahler, A. H., 1985. Geometric-optical modeling of a conifer forest canopy. Geoscience and Remote Sensing, IEEE Transactions on (5), pp. 705-721.

Li, X. and Strahler, A. H., 1992. Geometric-optical bidirectional reflectance modeling of the discrete crown vegetation canopy: Effect of crown shape and mutual shadowing. Geoscience and Remote Sensing, IEEE Transactions on 30(2), pp. 276-292.

Lio, A.; Ito, A., 2014. A Global Database of Field-observed Leaf Area Index in Woody Plant Species, 1932-2011.

Mishra, N., Haque, M. O., Leigh, L., Aaron, D., Helder, D. and Markham, B., 2014. Radiometric Cross Calibration of Landsat 8 Operational Land Imager (OLI) and Landsat 7 Enhanced Thematic Mapper Plus (ETM+). Remote Sensing 6(12), pp. 12619-12638

Nakaji, T., Ide, R., Takagi, K., Kosugi, Y., Ohkubo, S., Nasahara, K. N., Saigusa, N. and Oguma, H., 2008. Utility of spectral vegetation indices for estimation of light conversion efficiency in coniferous forests in Japan. agricultural and forest meteorology 148(5), pp. 776-787.

Rieg, L., Wichmann, V., Rutzinger, M., Sailer, R., Geist, T. and Stötter, J., 2014. Data infrastructure for multitemporal airborne lidar point cloud analysis-examples from physical geography in high mountain environments. Computers, Environment and Urban Systems 45, pp. 137-146.

Roberts, D. A., Gardner, M., Church, R., Ustin, S., Scheer, G. and Green, R., 1998. Mapping chaparral in the santa monica mountains using multiple endmember spectral mixture models. Remote Sensing of Environment 65(3), pp. 267-279.

Rouse, J., Haas, R., Schell, J., Deering, D. and Harlan, J., 1974. Monitoring the vernal advancements and retrogradation of natural vegetation. NASA/GSFC, Final Report, Greenbelt, MD, USA pp. 1-137.

Suits, G. H., 1973. The cause of azimuthal variations in directional reflectance of vegetative canopies. Remote Sensing of Environment 2, pp. 175-182.

Sukuvaara, T., Pulliainen, J., Kyrö, E., Suokanerva, H., Heikkinen, P. and Suomalainen, J., 2007. Reflectance spectroradiometer measurement system in 30 meter mast for validating satellite images. In: Geoscience and Remote Sensing Symposium, 2007. IGARSS 2007. IEEE International, IEEE, pp. 2885-2889.

United States Geological Survey, 2015a. Landsat 8 (L8) Data Users Handbook. United States Geological Survey.

United States Geological Survey, 2015b. Provisional Landsat 8 Surface Reflectance Product Guide.

United States Geological Survey, 2016. United States Geological Survey Earth Resources and Observation Science (EROS) Center Science Processing Architecture. https://espa.cr . usgs.gov/login/?next=/. Accessed: 2016-04-10.

Wallace, L., Lucieer, A., Watson, C. and Turner, D., 2012. Development of a uav-lidar system with application to forest inventory. Remote Sensing 4(6), pp. 1519-1543.

Zarco-Tejada, P. J., Berjón, A., López-Lozano, R., Miller, J., Martín, P., Cachorro, V., González, M. and De Frutos, A., 2005. Assessing vineyard condition with hyperspectral indices: Leaf and canopy reflectance simulation in a rowstructured discontinuous canopy. Remote Sensing of Environment 99(3), pp. 271-287.

Zhang, L., Sun, X., Wu, T. and Zhang, H., 2015. An Analysis of Shadow Effects on Spectral Vegetation Indexes Using a Ground-Based Imaging Spectrometer. Geoscience and Remote Sensing Letters, IEEE 12(11), pp. 2188-2192. 